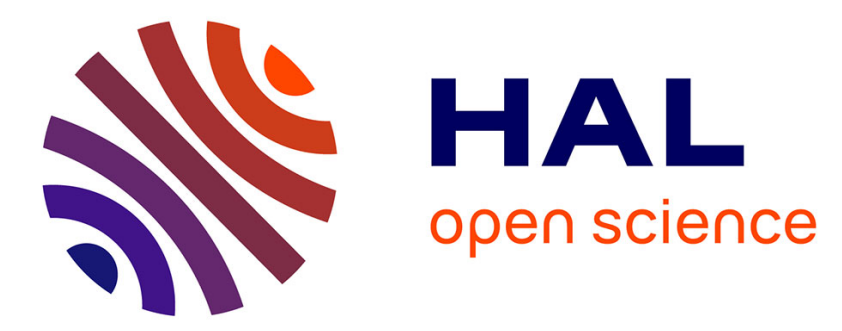

\title{
Les Chênes sessile et pédonculé (Quercus petraea Liebl. et Quercus robur L.) dans le réseau Renecofor : rythme de croissance radiale, anatomie du bois, de l'aubier et de l'écorce.
}

François Lebourgeois

\section{To cite this version:}

François Lebourgeois. Les Chênes sessile et pédonculé (Quercus petraea Liebl. et Quercus robur L.) dans le réseau Renecofor: rythme de croissance radiale, anatomie du bois, de l'aubier et de l'écorce.. Revue forestière française, 1999, 51 (4), pp.522-536. 10.4267/2042/5460 . hal-03443496

\author{
HAL Id: hal-03443496 \\ https://hal.science/hal-03443496
}

Submitted on 23 Nov 2021

HAL is a multi-disciplinary open access archive for the deposit and dissemination of scientific research documents, whether they are published or not. The documents may come from teaching and research institutions in France or abroad, or from public or private research centers.
L'archive ouverte pluridisciplinaire $\mathbf{H A L}$, est destinée au dépôt et à la diffusion de documents scientifiques de niveau recherche, publiés ou non, émanant des établissements d'enseignement et de recherche français ou étrangers, des laboratoires publics ou privés. 


\title{
LES CHÊNES SESSILE ET PÉDONCULÉ (Quercus petraea Liebl. et Quercus robur L.) DANS LE RÉSEAU RENECOFOR : RYTHME DE CROISSANCE RADIALE, ANATOMIE DU BOIS, DE L'AUBIER ET DE L'ÉCORCE
}

\author{
F. LEBOURGEOIS
}

Dans le cadre Réseau national de Suivi à long terme des Écosystèmes forestiers (RENECOFOR), l'étude dendrochronologique des 102 placettes d'observation (Lebourgeois, 1997), qui a retracé l'histoire des peuplements à travers la croissance radiale des arbres, a déjà fourni des informations pratiques pour les gestionnaires du réseau. Ainsi, la prise en compte du passé sylvicole des peuplements et des conditions climatiques locales a permis d'appréhender la réponse des arbres aux extrêmes climatiques (fortes réductions de croissance liées aux sécheresses ou aux froids hivernaux) et la durabilité des éclaircies pratiquées dans le peuplement. La détermination exacte de l'âge des arbres (à hauteur de carottage) a également permis de poser le problème de l'estimation des âges des peuplements sur des critères purement dendrométriques (hauteur et diamètre) (Lebourgeois et al., 1998).

Face à l'importance économique du Chêne en France et en raison de sa forte représentativité dans le réseau, il est apparu important d'analyser plus finement les caractéristiques dendrométriques et de croissance des 9 placettes de Chêne pédonculé (Quercus robur L.) et des 21 placettes de Chêne sessile [Quercus petraea (Matt.) Liebl]. Ainsi, à partir des carottes de sondage, nous avons pu analyser:

- le rythme de croissance radiale des peuplements,

- les caractéristiques et les variations de deux des composantes de la structure intrinsèque du bois (largeur et proportion du bois initial et du bois final),

- les caractéristiques de l'aubier et de l'écorce.

Les mesures concernant le bois initial et le bois final sont intéressantes, non seulement du point de vue de l'analyse du fonctionnement physiologique de l'arbre (Cochard et Tyree, 1990), mais également pour leur impact sur la qualité technologique des bois (Nepveu, 1990 ; Huber, 1993). En ce qui concerne l'aubier, l'intérêt de sa mesure est multiple. D'un point de vue hydrique, il joue un rôle fondamental dans les mouvements de l'eau dans l'arbre (Granier et al., 1994) et, d'un point de vue dendrométrique, la relation entre la croissance et la section d'aubier est un bon indicateur de la 
capacité photosynthétique et de la vitalité d'un arbre en peuplement (Bréda et al., 1994). Du point de vue sylvicole, les mesures de l'aubier permettent de mieux appréhender le volume utilisable par le scieur car, sa durabilité étant bien moindre que celle du duramen, il est généralement purgé au moment de l'exploitation des grumes (Polge, 1984). Le pourcentage moyen d'écorce est également un paramètre important à connaître pour le scieur car l'écorce constitue, dans la majorité des cas, un déchet qui ne l'intéresse pas.

Les objectifs de cette étude sont, d'une part, d'apporter quelques éléments d'information aux gestionnaires sur la croissance des chênaies du réseau et, d'autre part, de comparer les deux espèces de Chênes vis-à-vis des différents paramètres anatomiques et de croissance mesurés. Ce travail n'ayant pas pour objectifs une étude des relations station-production, nous n'avons pas cherché à analyser finement l'effet des facteurs stationnels dans les variations de ces paramètres et sur la productivité des peuplements. Seule la qualité de l'alimentation locale en eau, à travers le calcul de la réserve utile maximale du sol, a été prise en compte comme variable potentiellement explicative de la croissance.

\section{MATÉRIEL ET MÉTHODES}

Les protocoles d'installation des placettes d'échantillonnage des arbres dominants sondés par site pour l'étude dendrochronologique et des mesures des accroissements radiaux ont été développés dans des publications précédentes et ne seront donc pas repris ici (Ulrich, 1995 ; ONF, 1996 ; Lebourgeois, 1997).

Le comptage et la mesure des 73215 cernes provenant des 877 carottes de sondage (263 Chênes pédonculés et 614 Chênes sessiles) ont permis de déterminer pour chaque arbre :

- l'âge en 1994 (date du dernier cerne complet pris en compte),

- le niveau de croissance (sur différentes périodes et en fonction de l'âge des cernes),

- le temps de passage (en années) au pas de $5 \mathrm{~cm}$ d'accroissement du diamètre sous écorce (Pardé et Bouchon, 1988). Il est à noter que les temps de passage apportent des informations sur l'évolution du peuplement sur pied actuel, mais ne sont pas forcément représentatifs du peuplement ancien.

À partir des mesures brutes (précision 1/100 mm), la proportion de bois initial (zone poreuse à gros vaisseaux) et de bois final (zone à petits vaisseaux) a été déterminée pour chaque cerne en faisant le rapport entre les largeurs correspondantes et la largeur totale.

En ce qui concerne l'aubier, la limite entre le bois de cœur et l'aubier a été notée en observant chaque carotte juste après extraction du tronc (différence de couleur et d'humidité). La distance entre l'écorce et cette limite a été mesurée en $\mathrm{mm}$. À partir de cette largeur, le nombre de cernes dans l'aubier, sa surface (en $\mathrm{cm}^{2}$ ) et sa largeur relative (en \%) dans le tronc (à hauteur de carottage) ont été calculés pour chaque arbre.

Le pourcentage moyen d'écorce à hauteur de carottage a été calculé pour chaque arbre selon le protocole du Centre technique du Bois et de l'Ameublement en faisant le rapport entre le diamètre mesuré sur écorce et celui calculé sous écorce à partir des carottes de sondage prélevées au même niveau.

Pour chacun de ces paramètres, les caractéristiques du peuplement ont été obtenues en faisant la moyenne des mesures individuelles. La prise en compte de la moyenne ainsi que le nombre important d'arbres échantillonnés par site (23 à 30) et pour les deux espèces permettent de minimiser l'effet de la dissymétrie des arbres sur la valeur individuelle de ces paramètres (prélèvement d'une carotte unique à cœur entre 1 et $1,30 \mathrm{~m}$ ). 


\section{F. LEBOURGEOIS}

La gestion sylvicole des peuplements peut être appréhendée globalement à partir des informations disponibles à l'échelle de la parcelle (dates, nombres et volumes des coupes). Bien qu'importantes à connaître, ces informations sont cependant insuffisantes si l'on cherche à relier l'intensité de la compétition dans le peuplement au niveau de croissance radiale. Des travaux récents menés sur le Hêtre et le Chêne (Dhôte, 1995) montrent que l'effet de la sylviculture sur la croissance peut être étudié en reliant la croissance actuelle du peuplement à un indice de densité (Relative Density Index, RDI). Cet indice est calculé à partir de la circonférence quadratique moyenne du peuplement

Tableau I Comparaison des temps moyens de passage (au pas de $5 \mathrm{~cm}$ d'accroissement du diamètre sous écorce) et de la largeur moyenne du cerne annuel (en $\mathrm{mm}$ ) à différents âges cambiaux

et sur différentes périodes pour les 9 placettes de Chêne pédonculé (CHP) du réseau RENECOFOR

L'âge cambial est l'âge de l'arbre au moment où le cerne a été élaboré. Le chiffre après le code de l'espèce indique le département d'échantillonnage du peuplement. TSF $=$ taillis-sous-futaie ; $F=$ futaie

Le type de peuplement et le nombre d'interventions sylvicoles pratiquées dans la parcelle proviennent des informations récoltées par Ponce et al. (1998) sur l'historique des peuplements.

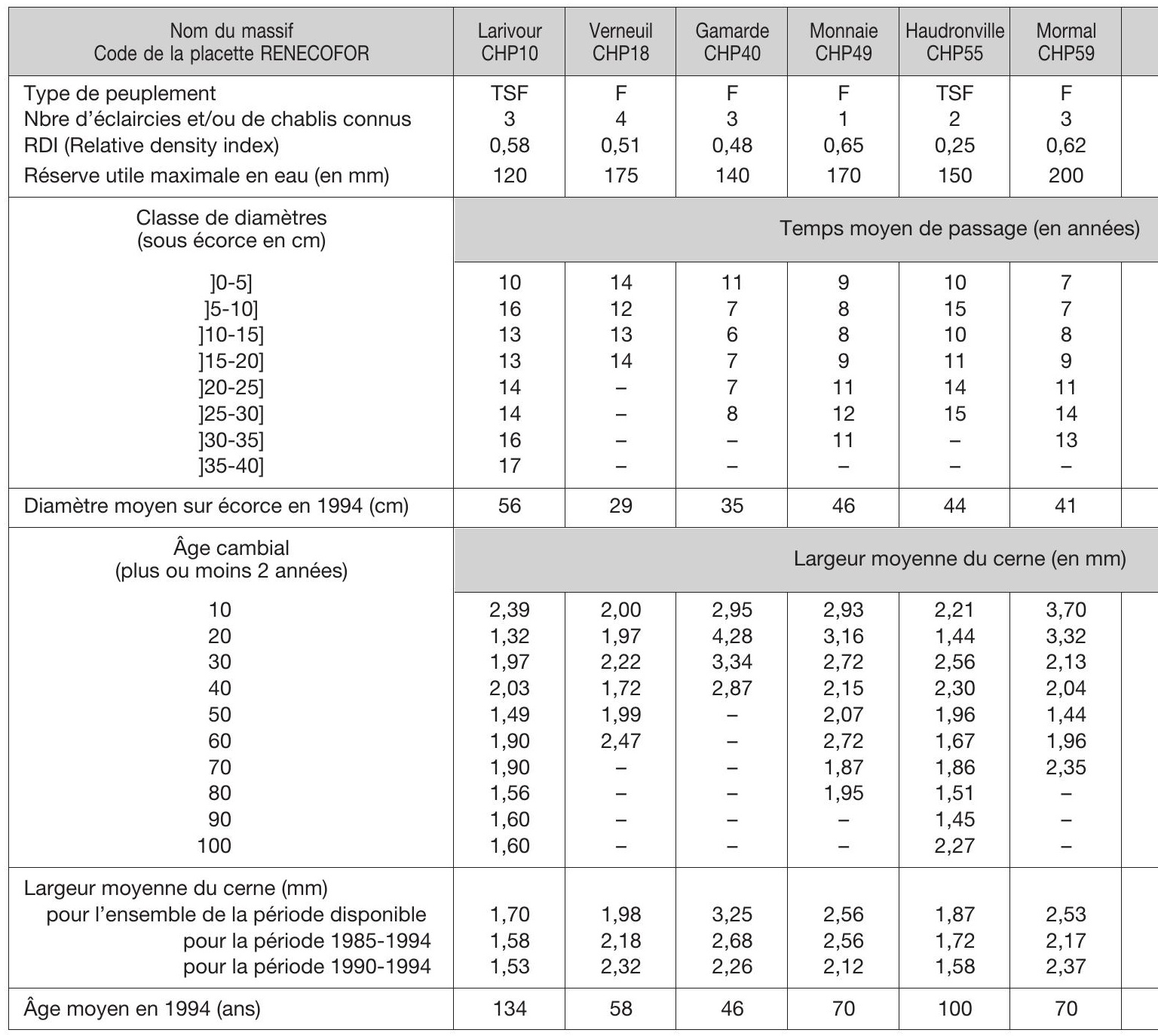


et du nombre de tiges à l'hectare (étage principal dominant). Sa valeur tend vers 0 quand les densités deviennent très faibles (faible compétition entre arbres). La valeur 1 correspond à la densité maximale pour laquelle des phénomènes "d'autoéclaircie" entrent en jeu. Pour les placettes du RENECOFOR, les indices de densité RDI ont été calculés par Cluzeau et al. (1998).

Pour chaque site, la réserve utile maximale en eau du sol (RUM exprimée en $\mathrm{mm}$ ) a été estimée à partir de la texture et de la charge en cailloux des divers horizons dans la limite de la profondeur prospectée par les racines (Brêthes et Ulrich, 1997).

L'analyse des effets de la RUM et de la densité du peuplement sur la croissance ne peut se faire que si l'on élimine préalablement (ou tout du moins que l'on minimise) les effets des autres paramètres influençant également le niveau de croissance des arbres, c'est-à-dire l'âge, le climat... Ceci complique les analyses et rend impossible la prise en compte simultanée de l'ensemble des sites disponibles. Ainsi, dans la majorité des cas, les analyses n'ont porté que sur des sous-échantillons de données présentant des caractéristiques comparables : même espèce, âge constant, largeurs de cernes fixées, années identiques...

\begin{tabular}{|c|c|c|}
\hline $\begin{array}{l}\text { Azereix } \\
\text { CHP65 }\end{array}$ & $\begin{array}{l}\text { Anjeux } \\
\text { CHP70 }\end{array}$ & $\begin{array}{c}\text { Pourlans } \\
\text { CHP71 }\end{array}$ \\
\hline $\mathrm{F}$ & $\mathrm{F}$ & $\mathrm{F}$ \\
\hline 3 & 2 & 6 \\
\hline 0,44 & 0,63 & 0,57 \\
\hline 140 & 170 & 180 \\
\hline 11 & 11 & 10 \\
\hline 11 & 9 & 9 \\
\hline 10 & 13 & 8 \\
\hline 10 & - & 10 \\
\hline 12 & - & 11 \\
\hline- & - & 14 \\
\hline- & - & - \\
\hline- & - & - \\
\hline 31 & 20 & 36 \\
\hline 2,35 & 2,38 & 2,64 \\
\hline 2,31 & 2,67 & 3,03 \\
\hline 2,47 & 2,36 & 2,68 \\
\hline 2,68 & 2,02 & 2,44 \\
\hline 2,50 & - & 2,17 \\
\hline 2,63 & - & 2,08 \\
\hline- & - & 1,91 \\
\hline- & - & - \\
\hline- & - & - \\
\hline- & - & - \\
\hline 2,41 & 2,39 & 2,46 \\
\hline 2,41 & 2,24 & 2,22 \\
\hline 2,19 & 1,97 & 1,79 \\
\hline 54 & 35 & 67 \\
\hline
\end{tabular}

La comparaison entre les deux espèces effectuée au niveau global a été complétée par une analyse détaillée du peuplement mélangé de la forêt de Fontainebleau (CPS 77). Cette placette est particulièrement intéressante pour une telle comparaison car elle présente, en mélange pied à pied, des Chênes sessile et pédonculé d'âge et de diamètre comparables ayant bénéficié des mêmes conditions stationnelles de croissance tout au long de leur vie (mêmes sol, climat et sylviculture). Les conditions écologiques de croissance des arbres étant en tout point comparables, l'expression de l'effet espèce sur les paramètres mesurés peut être étudiée en éliminant les effets de la station; effets potentiels non pris en compte par l'analyse globale.

\section{RÉSULTATS ET DISCUSSION}

\section{Rythme de croissance et temps de passage}

La dynamique de croissance radiale ainsi que son niveau diffèrent notablement entre les peuplements (tableaux I, p. 524 et II, p. 526). Dans le jeune âge, il faut de 7 à 19 ans pour passer la première classe de diamètre $] 0-5 \mathrm{~cm}]$. Par la suite, le temps moyen de passage diminue (forêt de Cîteaux ; CHS 21), augmente (forêt de Mouterhouse ; CHS 57b) ou se stabilise (forêt de Rennes ; CHS 35) selon le niveau de croissance. L'accroissement radial moyen, calculé sur l'ensemble de la vie du peuplement, varie de 1,51 mm pour le peuplement âgé (137 ans) de Chêne sessile de la Hardt (CHS 68) à $3,25 \mathrm{~mm}$ pour la jeune futaie (46 ans) de Chêne pédonculé de la forêt de Gamarde (CHP 40).

Pour le Chêne pédonculé, les objectifs sylvicoles actuels sont de produire du bois de gros diamètre (supérieur à $70 \mathrm{~cm}$ ) avec des cernes réguliers de 2,5 à 4 mm (âge d'exploitabilité de 100 à 140 ans) (Duplat, 1996). Pour le Chêne sessile, la largeur de cerne souhaitée varie de 2 à $2,5 \mathrm{~mm}$ pour un âge d'exploitabilité de 170 à 205 ans (Jarret, 1996). Par rapport à ces "normes", il apparaît donc que les peuplements de Chêne pédonculé ont 


\section{F. LEBOURGEOIS}

Tableau II

Comparaison des temps moyens de passage

(au pas de $5 \mathrm{~cm}$ d'accroissement du diamètre sous écorce)

et de la largeur moyenne du cerne annuel (en $\mathrm{mm}$ )

à différents âges cambiaux et sur différentes périodes pour les 21 placettes de Chêne sessile (CHS) du réseau RENECOFOR

\begin{tabular}{|c|c|c|c|c|c|c|c|c|c|}
\hline $\begin{array}{l}\text { Nom du massif } \\
\text { Code de la placette } \\
\text { RENECOFOR }\end{array}$ & $\begin{array}{l}\text { Seillon } \\
\text { CHSO1 }\end{array}$ & $\begin{array}{l}\text { Tronçais } \\
\text { CHSO3 }\end{array}$ & $\begin{array}{l}\text { Temple } \\
\text { CHS10 }\end{array}$ & $\begin{array}{l}\text { Vierzon } \\
\text { CHS18 }\end{array}$ & $\begin{array}{l}\text { Citeaux } \\
\text { CHS21 }\end{array}$ & $\begin{array}{l}\text { Lyons } \\
\text { CHS27 }\end{array}$ & $\begin{array}{l}\text { Rennes } \\
\text { CHS35 }\end{array}$ & $\begin{array}{l}\text { Blois } \\
\text { CHS41 }\end{array}$ & $\begin{array}{c}\text { Chatrices } \\
\text { CHS51 }\end{array}$ \\
\hline Type de peuplement & $\mathrm{F}$ & $\mathrm{F}$ & $\mathrm{F}$ & $\mathrm{F}$ & $\mathrm{F}$ & $\mathrm{F}$ & $\mathrm{F}$ & $\mathrm{F}$ & TSF \\
\hline Nbre d'éclaircies et/ou de chablis connus & 5 & 6 & 4 & 9 & 4 & 4 & 11 & 6 & 5 \\
\hline RDI (Relative density index) & 0,60 & 0,77 & 0,53 & 0,56 & 0,63 & 0,61 & 0,75 & 0,69 & 0,62 \\
\hline Réserve utile maximale en eau (en mm) & 170 & 75 & 200 & 130 & 200 & 170 & 120 & 175 & 55 \\
\hline $\begin{array}{l}\text { Classe de diamètres } \\
\text { (sous écorce en } \mathrm{cm} \text { ) }\end{array}$ & \multicolumn{9}{|c|}{ Temps moyen } \\
\hline ]0-5] & 15 & 14 & 15 & 13 & 14 & 8 & 12 & 11 & 11 \\
\hline ]5-10] & 13 & 14 & 13 & 11 & 14 & 11 & 12 & 14 & 20 \\
\hline ]10-15] & 14 & 12 & 13 & 9 & 13 & 9 & 12 & 12 & 14 \\
\hline ]15-20] & 13 & 16 & 12 & 10 & 10 & 10 & 13 & 12 & 16 \\
\hline ]20-25] & 14 & 17 & 11 & 9 & 10 & 10 & 13 & 12 & 15 \\
\hline ]25-30] & 13 & 15 & 17 & 11 & 11 & - & 13 & 12 & 19 \\
\hline ]30-35] & - & 15 & - & 11 & 9 & - & 11 & 14 & 15 \\
\hline ]35-40] & - & - & - & - & - & - & 12 & - & 15 \\
\hline Diamètre moyen sur écorce en $1994(\mathrm{~cm})$ & 40 & 47 & 36 & 43 & 44 & 30 & 47 & 42 & 53 \\
\hline $\begin{array}{c}\text { Âge cambial } \\
\text { (plus ou moins } 2 \text { années) }\end{array}$ & \multicolumn{9}{|c|}{ Largeur moyenne } \\
\hline 10 & 1,66 & 1,80 & 1,69 & 1,96 & 1,79 & 2,79 & 2,00 & 2,02 & 1,68 \\
\hline 20 & 1,92 & 1,85 & 1,94 & 2,40 & 1,81 & 2,37 & 2,18 & 1,9 & 1,13 \\
\hline 30 & 1,84 & 2,01 & 2,05 & 2,72 & 1,67 & 2,86 & 2,19 & 2,09 & 1,89 \\
\hline 40 & 1,9 & 2,04 & 1,90 & 2,65 & 2,38 & 2,36 & 1,86 & 2,05 & 1,67 \\
\hline 50 & 1,92 & 1,51 & 2,16 & 2,59 & 2,66 & 2,56 & 1,94 & 2,02 & 1,52 \\
\hline 60 & 1,78 & 1,45 & 2,29 & 2,08 & 2,31 & - & 1,83 & 2,18 & 1,67 \\
\hline 70 & 1,81 & 1,41 & 2,09 & 2,52 & 2,43 & - & 2,03 & 2,10 & 1,56 \\
\hline 80 & 2,24 & 1,63 & 2,21 & - & 3,01 & - & 2,15 & 2,00 & 1,14 \\
\hline 90 & 2,31 & 1,65 & - & - & - & - & 2,02 & 2,11 & 1,50 \\
\hline 100 & - & 1,59 & - & - & - & - & 2,13 & - & 1,72 \\
\hline $\begin{array}{l}\text { Largeur moyenne du cerne }(\mathrm{mm}) \\
\text { pour l'ensemble de la période disponible }\end{array}$ & 1,90 & 1,70 & 2,05 & 2,39 & 2,25 & 2,58 & 2,04 & 2,05 & 1,59 \\
\hline pour la période 1985-1994 & 2,43 & 1,72 & 2,78 & 2,44 & 2,97 & 2,52 & 2,35 & 2,07 & 1,87 \\
\hline pour la période 1990-1994 & 2,34 & 1,74 & 2,76 & 2,25 & 2,71 & 2,65 & 2,45 & 1,90 & 1,84 \\
\hline Âge en 1994 (ans) & 88 & 115 & 83 & 78 & 87 & 55 & 101 & 92 & 139 \\
\hline
\end{tabular}

une croissance actuelle plus faible que celle recommandée, et que la croissance de la majorité des placettes de Chêne sessile est assez similaire voire supérieure aux objectifs. Bien qu'intéressants à connaître pour le gestionnaire, ces résultats globaux doivent être considérés avec précaution. En effet, les objectifs sylvicoles dépendant de la fertilité de la station et de la sylviculture 


\section{Biologie et forêt}

L'âge cambial est l'âge de l'arbre au moment où le cerne a été élaboré. Le chiffre après le code de l'espèce indique le département d'échantillonnage du peuplement. TSF = taillis-sous-futaie $; \mathrm{F}=$ futaie

Le type de peuplement et le nombre d'interventions sylvicoles pratiquées dans la parcelle proviennent des informations récoltées par Ponce et al. (1998) sur l'historique des peuplements. CPS = mélange de Chênes sessile et pédonculé.

\begin{tabular}{|c|c|c|c|c|c|c|c|c|c|c|c|}
\hline $\begin{array}{c}\text { Amelecourt } \\
\text { CHS57a }\end{array}$ & $\begin{array}{c}\text { Mouterhouse } \\
\text { CHS57b }\end{array}$ & $\begin{array}{l}\text { Vincence } \\
\text { CHS58 }\end{array}$ & $\begin{array}{l}\text { Hez- } \\
\text { Froidmont } \\
\text { CHS60 }\end{array}$ & $\begin{array}{l}\text { Réno- } \\
\text { Valdieu } \\
\text { CHS61 }\end{array}$ & $\begin{array}{c}\text { Nonnenhardt } \\
\text { CHS67 }\end{array}$ & $\begin{array}{l}\text { Hardt } \\
\text { CHS68 }\end{array}$ & $\begin{array}{l}\text { Bercé } \\
\text { CHS72 }\end{array}$ & $\begin{array}{c}\text { Fontainebleau } \\
\text { CPS77 }\end{array}$ & $\begin{array}{l}\text { Grésigne } \\
\text { CHS81 }\end{array}$ & $\begin{array}{l}\text { Moulière } \\
\text { CHS86 }\end{array}$ & $\begin{array}{l}\text { Darney } \\
\text { CHS88 }\end{array}$ \\
\hline$F$ & $\mathrm{~F}$ & $\mathrm{~F}$ & $\mathrm{~F}$ & $\mathrm{~F}$ & $\mathrm{~F}$ & TSF & $\mathrm{F}$ & $\mathrm{F}$ & $\mathrm{F}$ & $\mathrm{F}$ & $\mathrm{F}$ \\
\hline 5 & 6 & 3 & 4 & 4 & 13 & 7 & 4 & 8 & 8 & 6 & 8 \\
\hline 0,51 & 0,66 & 0,81 & 0,57 & 0,78 & 0,65 & 0,44 & 0,79 & 0,44 & 0,64 & 0,69 & 0,48 \\
\hline 140 & 80 & 150 & 125 & 110 & 100 & 85 & 135 & 135 & 85 & 80 & 185 \\
\hline \multicolumn{12}{|c|}{ de passage (en années) } \\
\hline 10 & 10 & 12 & 10 & 14 & 11 & 13 & 13 & 19 & 12 & 15 & 17 \\
\hline 11 & 12 & 13 & 8 & 16 & 16 & 19 & 11 & 12 & 12 & 13 & 16 \\
\hline 11 & 15 & 11 & 9 & 13 & 14 & 14 & 10 & 11 & 16 & 15 & 16 \\
\hline 13 & 17 & 9 & 10 & 15 & 11 & 14 & 9 & 14 & 18 & 12 & 16 \\
\hline 12 & 19 & 11 & 10 & 15 & 12 & 15 & 11 & 15 & 16 & 11 & 20 \\
\hline 13 & 21 & - & 11 & 15 & - & 16 & - & 15 & 17 & 15 & 17 \\
\hline 11 & 17 & - & - & - & - & - & - & 16 & - & - & 16 \\
\hline- & - & - & - & - & - & - & - & - & - & - & - \\
\hline 41 & 45 & 31 & 36 & 36 & 34 & 47 & 34 & 46 & 38 & 37 & 44 \\
\hline \multicolumn{12}{|c|}{ du cerne (en mm) } \\
\hline 2,16 & 2,36 & 1,87 & 2,49 & 1,55 & 1,97 & 1,72 & 2,01 & 1,18 & 1,94 & 1,57 & 1,31 \\
\hline 2,61 & 2,23 & 2,01 & 2,84 & 1,68 & 1,53 & 1,19 & 2,38 & 1,69 & 1,99 & 1,94 & 1,45 \\
\hline 1,90 & 1,70 & 2,30 & 2,71 & 1,70 & 1,56 & 1,48 & 2,41 & 2,41 & 1,65 & 1,70 & 1,58 \\
\hline 2,09 & 1,57 & 2,71 & 2,42 & 1,93 & 2,02 & 1,87 & 2,61 & 2,08 & 1,56 & 1,64 & 1,68 \\
\hline 2,10 & 1,36 & 2,40 & 2,40 & 1,37 & 2,35 & 1,88 & 2,51 & 1,66 & 1,32 & 2,06 & 1,47 \\
\hline 2,26 & 1,26 & 2,35 & 2,71 & 1,87 & 2,08 & 1,50 & 2,34 & 1,68 & 1,42 & 2,38 & 1,56 \\
\hline 1,87 & 1,35 & - & - & 1,57 & 2,21 & 1,64 & - & 1,66 & 1,56 & 2,36 & 1,22 \\
\hline 2,55 & 1,16 & - & - & 1,94 & 2,06 & 1,49 & - & 1,71 & 1,47 & 2,24 & 1,35 \\
\hline- & 1,20 & - & - & - & - & 1,50 & - & 1,48 & 1,43 & - & 1,40 \\
\hline- & 1,45 & - & - & - & - & 1,50 & - & 1,85 & 1,62 & - & 1,57 \\
\hline 2,18 & 1,52 & 2,25 & 2,64 & 1,76 & 1,97 & 1,51 & 2,34 & 1,78 & 1,62 & 1,94 & 1,49 \\
\hline 2,49 & 1,36 & 2,46 & 2,73 & 2,03 & 2,22 & 1,58 & 2,32 & 2,20 & 1,47 & 2,31 & 1,95 \\
\hline 2,46 & 1,29 & 2,44 & 2,99 & 2,16 & 2,32 & 1,55 & 2,15 & 2,13 & 1,53 & 2,04 & 1,98 \\
\hline 85 & 128 & 61 & 60 & 88 & 76 & 137 & 64 & 113 & 98 & 82 & 129 \\
\hline
\end{tabular}

pratiquée dans le peuplement, la prise en compte de ces paramètres s'avère indispensable pour une analyse objective des niveaux de croissance des chênaies. Ainsi, pour le Chêne sessile, les niveaux de croissance les plus élevés correspondent aux sites bien alimentés en eau pour lesquels la compétition entre arbre est faible (figure 1, p. 528). 
Figure 1 RELATION ENTRE LA CROISSANCE RADIALE MOYENNE (en mm/an)

CALCULÉE SUR LA PÉRIODE 1985-1994, LA RÉSERVE UTILE MAXIMALE EN EAU DU SOL (en mm) (trait continu)

ET L'INDICE DE DENSITÉ (tireté) POUR 12 PEUPLEMENTS DE CHÊNE SESSILE DU RÉSEAU RENECOFOR

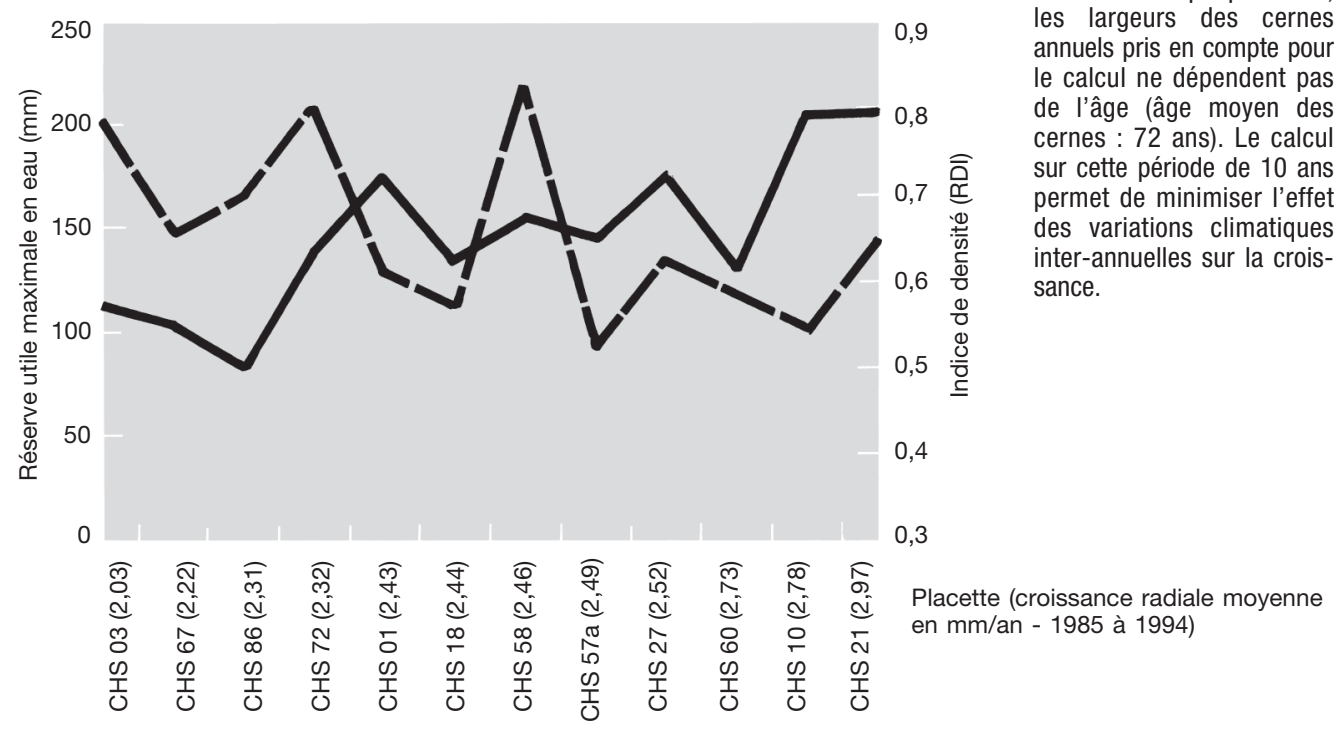

\section{Largeur et proportion du bois initial et du bois final dans les cernes annuels}

\section{- Relation avec l'âge cambial}

La largeur du bois initial augmente linéairement des jeunes cernes aux cernes âgés (figure 2, p. 529). Pour les Chênes sessiles, l'augmentation est de l'ordre de 0,35 \% par an dans la gamme d'âge cambial de 10 à 80 ans ; la largeur du bois initial étant de l'ordre de 0,4 mm à 10 ans. Bien qu'une diminution de la largeur du bois final avec l'âge soit observable pour les peuplements les plus âgés, il n'est pas possible de dégager, comme pour le bois initial, une tendance générale pour ce compartiment (figure 2). En ce qui concerne les proportions des deux compartiments, le pourcentage de bois initial augmente des jeunes cernes (10 ans) aux cernes âgés (> 70 ans) dans des proportions de $20 \%$ à 30-35\% pour le Chêne sessile et jusqu'à 40-45\% pour le Chêne pédonculé. Pour les Chênes de la forêt de Fontainebleau, les deux espèces se différencient nettement à partir de 60-70 ans et, à 100 ans, la proportion de bois initial est de l'ordre de $38 \%$ pour le Chêne pédonculé et de $30 \%$ pour le Chêne sessile (figure 3, p. 530).

Cette différence entre les deux espèces rejoint les observations faites par différents auteurs (voir synthèse dans Feuillat et al., 1997) et pourrait être liée, en partie, aux besoins en eau plus importants du Chêne pédonculé (Lévy et al., 1992) et expliquer sa plus grande vulnérabilité à la cavitation (Bréda et al., 1993). Ces différences pourraient également être partiellement mises en rapport avec celles observées dans l'aubier (voir paragraphe suivant). Le Chêne sessile "compenserait" une plus faible proportion de bois initial, c'est-à-dire de gros vaisseaux (potentiellement) conducteurs, par un plus grand nombre d'années d'aubier et donc une largeur d'aubier plus importante. Cette hypothèse doit cependant être modulée par des travaux récents qui montrent qu'une grande partie des vaisseaux de bois initial perd sa capacité de conduction au cours de l'hiver suite à des phénomènes d'embolie (Cochard et al., 1992) et, qu'au cours de la saison de croissance, ces vaisseaux ne sont fonctionnels que dans le cerne le plus externe (Granier et al., 1994). Ces mêmes auteurs ont toutefois montré qu'une détérioration du dernier cerne conduisait à une réactivation des cernes plus anciens. 
Figure 2

ÉVOLUTION DES LARGEURS DE BOIS INITIAL (BI : trait fin) ET FINAL (BF : trait épais)

EN FONCTION DE L'ÂGE CAMBIAL (âge de l'arbre au moment de l'élaboration du cerne) POUR 8 PEUPLEMENTS DE CHÊNES SESSILE (CHS) ET PÉDONCULÉ (CHP) DU RÉSEAU RENECOFOR

Pour chaque âge cambial et chaque placette,

la moyenne a été calculée sur au moins 18 cernes.

F. de Monnaie (CHP 49)

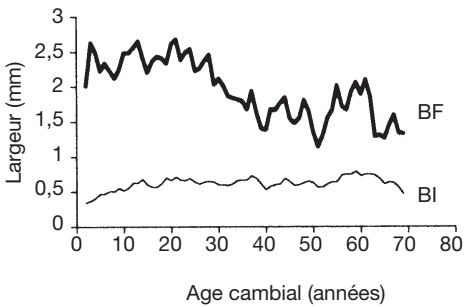

F. de Bercé (CHS 72)

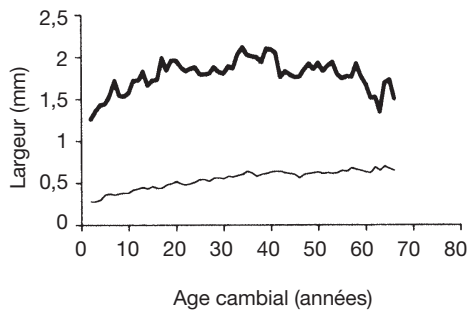

F. de Vierzon (CHS 18)
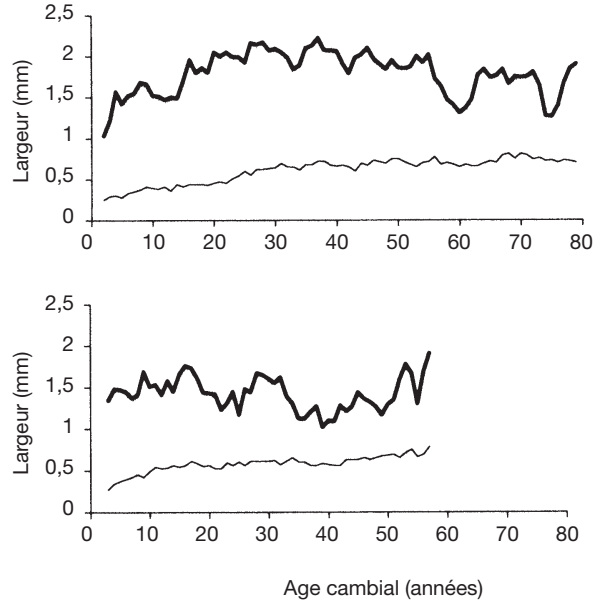

F. de Verneuil (CHP 18)

F. d'Azereix (CHP 65)

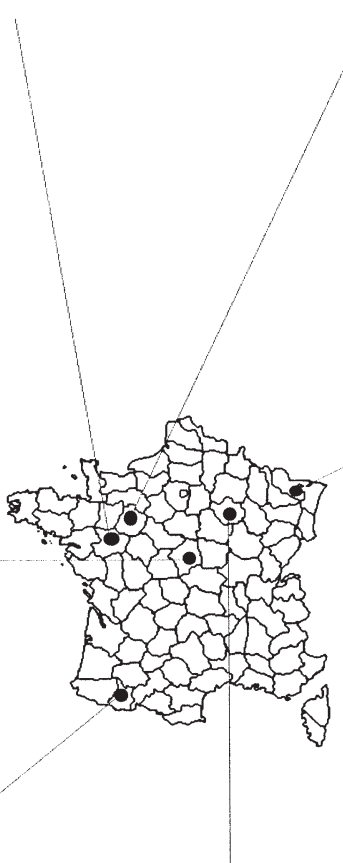

F. d'Amelecourt (CHS 57a)

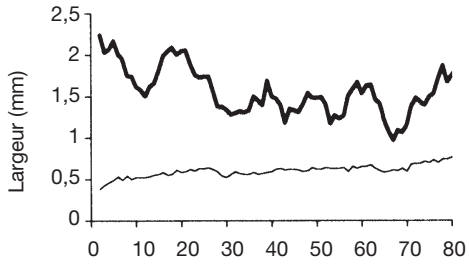

Age cambial (années)
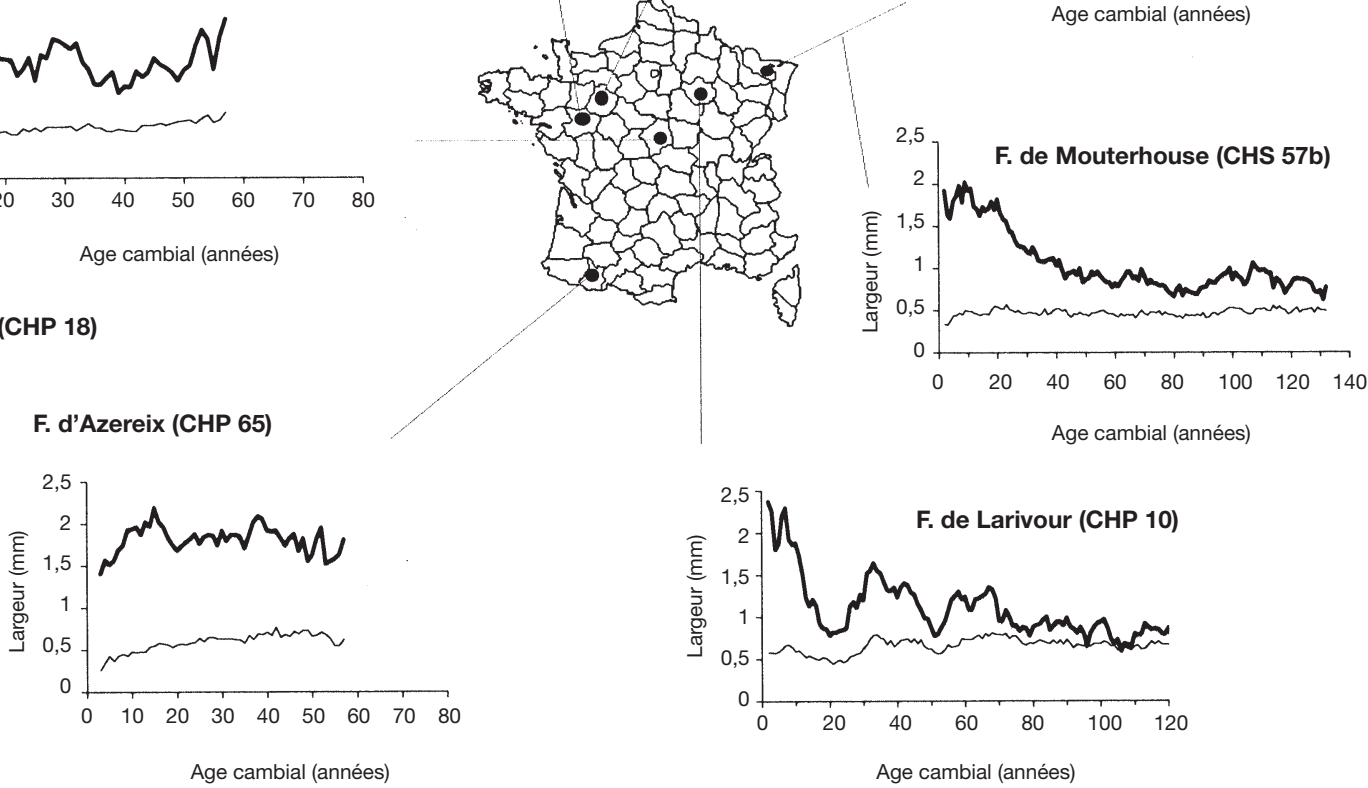

Age cambial (années) 


\section{F. LEBOURGEOIS}

Figure 3 ÉVOLUTION DE LA PROPORTION MOYENNE DE BOIS INITIAL (BI en \%) DANS LE CERNE ANNUEL SELON L'ÂGE CAMBIAL (âge de l'arbre au moment de l'élaboration du cerne)

POUR LES 12 CHÊNES SESSILES (rond blanc) ET LES 16 CHÊNES PÉDONCULÉS (rond noir) DE LA PLACETTE MIXTE (CPS 77) DE LA FORÊT DE FONTAINEBLEAU DU RÉSEAU RENECOFOR

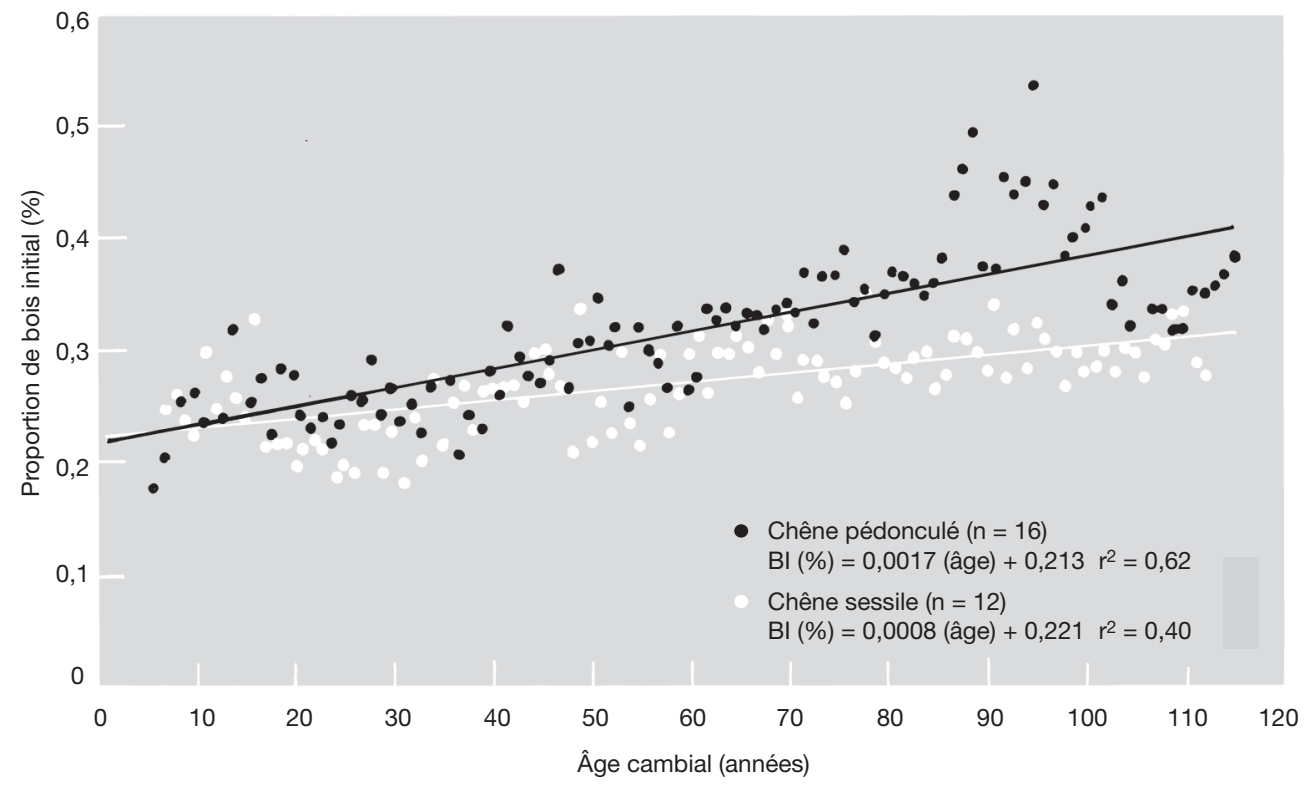

- Relation avec la largeur de cerne

À âge cambial fixé, les largeurs des deux compartiments augmentent parallèlement à la largeur totale du cerne annuel (tableau III, ci-dessous). À largeur du cerne fixée, la largeur du bois final tend à être moins importante chez le Chêne pédonculé. Pour les chênes de Fontainebleau, la différence

Tableau III Largeur (en mm) et proportion (en \%) du bois initial (Bl) et du bois final (BF) selon la largeur totale du cerne annuel (CC en $\mathrm{mm}$ ) d'âge cambial compris entre $\mathbf{4 0}$ et $\mathbf{6 0}$ ans Pour cette gamme d'âges, le nombre de cernes analysés est respectivement de 4224 et 12689 pour le Chêne pédonculé et sessile. Les valeurs indiquées ont été obtenues à partir des différentes équations de régression liant les deux compartiments au cerne total.

Pour le Chêne pédonculé : $B F(m m)=0,865(C C)-0,365\left(r^{2}=0,94\right)$ et $\% B F=0,171 \mathrm{Ln}(C C)+0,553\left(r^{2}=0,33\right)$. Pour le Chêne sessile : $B F(m m)=0,848(C C)-0,265\left(r^{2}=0,95\right)$ et $\% B F=0,127 \operatorname{Ln}(C C)+0,621\left(r^{2}=0,25\right)$.

Ces équations sont valides dans la gamme des largeurs de cerne total compris entre 1 et $8 \mathrm{~mm}$.

\begin{tabular}{|c|c|c|c|c|c|c|c|c|}
\hline \multirow{3}{*}{$\begin{array}{l}\text { Largeur } \\
\text { du cerne complet }\end{array}$} & \multicolumn{4}{|c|}{ Chêne pédonculé } & \multicolumn{4}{|c|}{ Chêne sessile } \\
\hline & \multicolumn{2}{|c|}{ Largeur (mm) } & \multicolumn{2}{|c|}{ Proportion (\%) } & \multicolumn{2}{|c|}{ Largeur (mm) } & \multicolumn{2}{|c|}{ Proportion (\%) } \\
\hline & $\mathrm{BI}$ & $\mathrm{BF}$ & $\mathrm{BI}$ & $\mathrm{BF}$ & $\mathrm{BI}$ & $\mathrm{BF}$ & $\mathrm{BI}$ & BF \\
\hline 0,5 & 0,25 & 0,25 & 0,47 & 0,53 & 0,24 & 0,26 & 0,43 & 0,57 \\
\hline $1 \ldots$ & 0,50 & 0,50 & 0,45 & 0,55 & 0,42 & 0,58 & 0,38 & 0,62 \\
\hline 1,5 & 0,57 & 0,93 & 0,38 & 0,62 & 0,50 & 1,00 & 0,33 & 0,67 \\
\hline 2. & 0,63 & 1,37 & 0,33 & 0,67 & 0,57 & 1,43 & 0,29 & 0,71 \\
\hline 2,5 & 0,70 & 1,80 & 0,29 & 0,71 & 0,65 & 1,85 & 0,26 & 0,74 \\
\hline $3 \ldots$ & 0,77 & 2,23 & 0,26 & 0,74 & 0,72 & 2,28 & 0,24 & 0,76 \\
\hline
\end{tabular}


apparaît nettement pour les cernes fins $(0,5$ et $1 \mathrm{~mm})$ avec respectivement des largeurs moyennes de bois final de 0,16 et 0,59 mm pour le Chêne pédonculé et de 0,28 et 0,69 pour le Chêne sessile. Pour les cernes de plus de $2 \mathrm{~mm}$, la largeur de bois final est identique pour les deux espèces (2,3 $\mathrm{mm}$ pour un cerne de $3 \mathrm{~mm}$ ). En ce qui concerne la proportion de bois final, une augmentation de la largeur du cerne de 1 à $3 \mathrm{~mm}$ se traduit par une augmentation relative de respectivement $23 \%$ et $35 \%$ pour le Chêne sessile et pédonculé (tableau III). Pour les deux espèces, l'augmentation de la proportion de bois final est très forte pour les cernes fins (environ $+17 \%$ pour une augmentation de la largeur de $0,5 \mathrm{~mm}$ à $1,5 \mathrm{~mm}$ ) puis se ralentit pour les cernes de plus en plus larges (environ $+3 \%$ pour un passage de 2,5 à $3 \mathrm{~mm}$ ). Du point de vue qualitatif, ceci signifie que la densité du bois augmente et donc que sa qualité technologique devient moindre (Polge et Keller, 1973 ; Zhang et al., 1993, 1994 ; Feuillat et al., 1997).

\section{Le bois d'aubier}

Les caractéristiques du bois d'aubier varient selon l'espèce et l'âge des arbres (tableau IV, cidessous). Globalement, l'aubier du Chêne pédonculé est de $15 \%$ à $20 \%$ moins large que celui du Chêne sessile, ce qui est cohérent avec les résultats obtenus par différents auteurs (tableau $\mathrm{V}$, p. 532). Cette différence n'est pas due à une croissance moyenne dans l'aubier supérieure (2,2 $\mathrm{mm}$ /an pour les deux espèces dans la gamme d'âges 50 à 90 ans) mais à un nombre de cernes dans l'aubier plus grand (14,5 cernes pour le Chêne sessile contre 13 pour le Chêne pédonculé). En moyenne, il apparait que la surface d'aubier tend à être plus élevée chez les arbres âgés et que la proportion relative dans le tronc diminue. La différence entre les deux espèces apparaît nettement dans la placette de Fontainebleau pour laquelle la surface d'aubier du Chêne sessile est de $35 \%$ plus importante que celle du Chêne pédonculé à âge et diamètre équivalents (tableau IV, ci-dessous).

Tableau IV

Caractéristiques de l'aubier par classes d'âges (à 1,30 m en 1994) pour les Chênes sessile (CHS) et pédonculé (CHP) du réseau RENECOFOR $\mathrm{n}=$ nombre d'arbres disponibles par classe d'âges.

Pour la classe d'âges [50 à 90 ans] et le peuplement de Fontainebleau, toutes les valeurs des paramètres caractérisant l'aubier sont significativement différentes au seuil de $5 \%$ entre les deux espèces (test $t$ ).

Pour les arbres plus âgés, seul le \% d'aubier est significativement différent.

\begin{tabular}{|c|c|c|c|c|c|}
\hline & $\begin{array}{l}\text { Nombre } \\
\text { de cerne }\end{array}$ & $\begin{array}{l}\text { Largeur } \\
(\mathrm{cm})\end{array}$ & $\begin{array}{c}\text { Surface } \\
\left(\mathrm{cm}^{2}\right)\end{array}$ & $\begin{array}{c}\text { Aubier } \\
(\%)\end{array}$ & $\begin{array}{l}\text { Diamètre } \\
(\mathrm{cm})\end{array}$ \\
\hline $\begin{array}{l}<50 \text { ans } \\
\text { CHP ( } n=62 ; 41 \text { ans) } \\
\text { CHS }\end{array}$ & $\begin{array}{l}9,8 \\
-\end{array}$ & $\begin{array}{c}2,4 \\
-\end{array}$ & $\begin{array}{c}167,7 \\
-\end{array}$ & $\begin{array}{c}20,9 \\
-\end{array}$ & $\begin{array}{c}27,5 \\
-\end{array}$ \\
\hline $\begin{array}{l}{[50 \text { à } 90 \text { ans }]} \\
\text { CHP ( } \mathrm{n}=164 ; 66 \text { ans) } \\
\text { CHS }(\mathrm{n}=349 ; 75 \text { ans })\end{array}$ & $\begin{array}{l}13,0 \\
14,5\end{array}$ & $\begin{array}{l}2,8 \\
3,4\end{array}$ & $\begin{array}{l}254,8 \\
313,3\end{array}$ & $\begin{array}{l}18,4 \\
21,1\end{array}$ & $\begin{array}{l}36,9 \\
36,8\end{array}$ \\
\hline $\begin{array}{l}190 \text { à } 120 \text { ans }] \\
\text { CHP } \\
\text { CHS ( } n=169 ; 105 \text { ans) }\end{array}$ & $\overline{15,8}$ & $\overline{3,0}$ & $\overline{3} \overline{6}, 9$ & $\overline{15,9}$ & $\overline{43,8}$ \\
\hline $\begin{array}{l}>120 \text { ans } \\
\text { CHP }(n=32 ; 145 \text { ans }) \\
\text { CHS }(n=94 ; 137 \text { ans })\end{array}$ & $\begin{array}{l}16,8 \\
17,3\end{array}$ & $\begin{array}{l}2,3 \\
2,7\end{array}$ & $\begin{array}{l}342,6 \\
329,4\end{array}$ & $\begin{array}{r}9,7 \\
12,9\end{array}$ & $\begin{array}{l}58,4 \\
47,8\end{array}$ \\
\hline $\begin{array}{l}\text { Forêt de Fontainebleau } \\
\text { CHP ( } n=16 ; 114 \text { ans) } \\
\text { CHS ( } n=12 ; 113 \text { ans) }\end{array}$ & $\begin{array}{l}14,5 \\
16,8\end{array}$ & $\begin{array}{l}2,8 \\
3,9\end{array}$ & $\begin{array}{l}326,9 \\
441,2\end{array}$ & $\begin{array}{l}13,9 \\
19,6\end{array}$ & $\begin{array}{l}45,1 \\
46,1\end{array}$ \\
\hline
\end{tabular}




\section{F. LEBOURGEOIS}

Tableau V

Caractéristiques moyennes du bois d'aubier (à 1,30 m) observées par différents auteurs pour les Chênes sessile et pédonculé

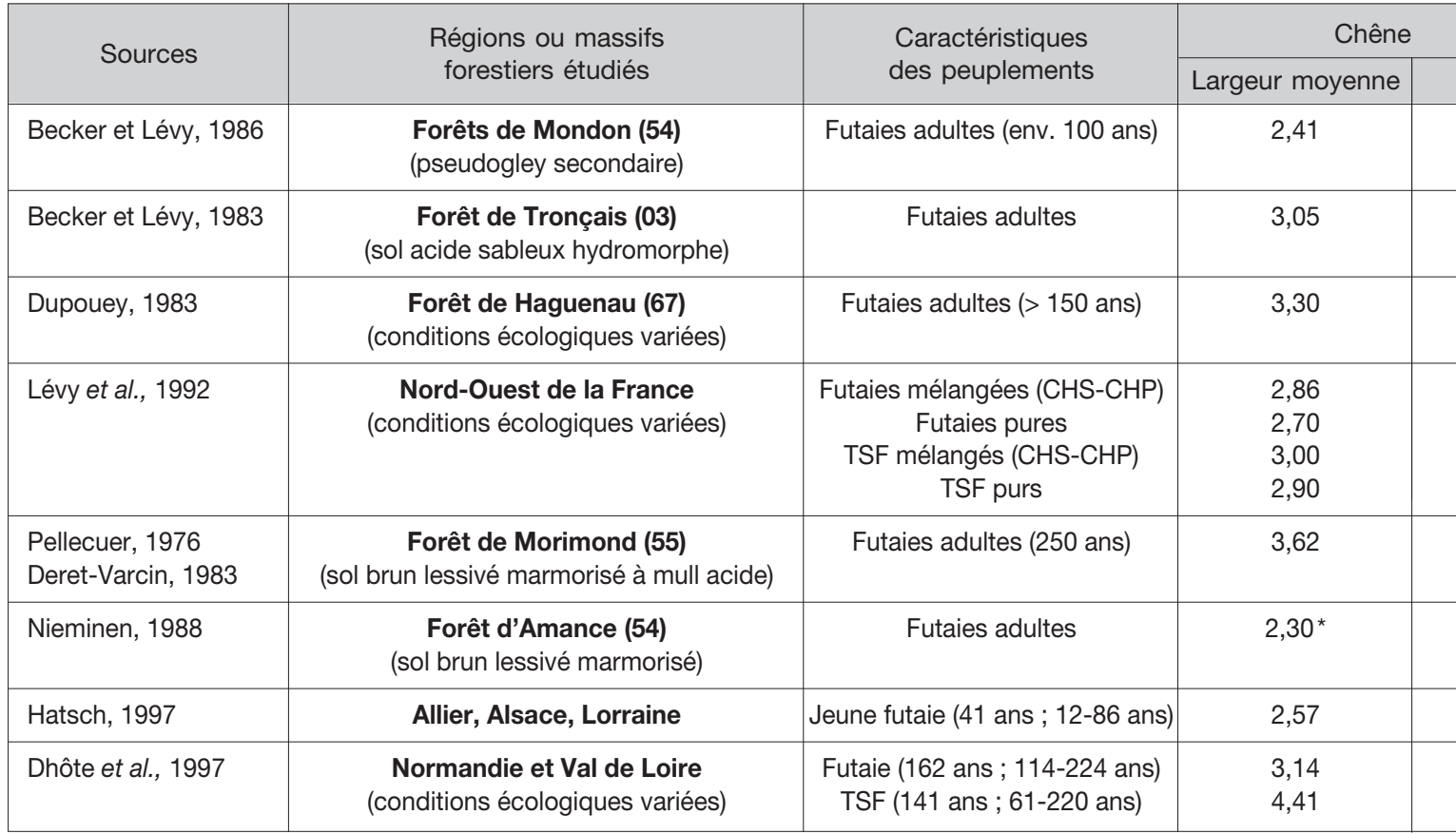

* valeurs obtenues sur des carottes prélevées à 2,80 m

TSF = taillis-sous-futaie ; CHS = Chêne sessile ; CHP = Chêne pédonculé.

Figure 4 RELATION ENTRE LA SURFACE D'AUBIER (à $1,30 \mathrm{~m}$ en $\mathrm{cm}^{2}$ ),

LE DIAMÈTRE SUR ÉCORCE (à 1,30 $\mathrm{m}$ en cm) ET LA RÉSERVE UTILE MAXIMALE EN EAU DU SOL (RUM en mm) POUR LES CHÊNES SESSILES ÂGÉS DE 75 À 85 ANS EN 1994 (âge moyen = 82 ans)

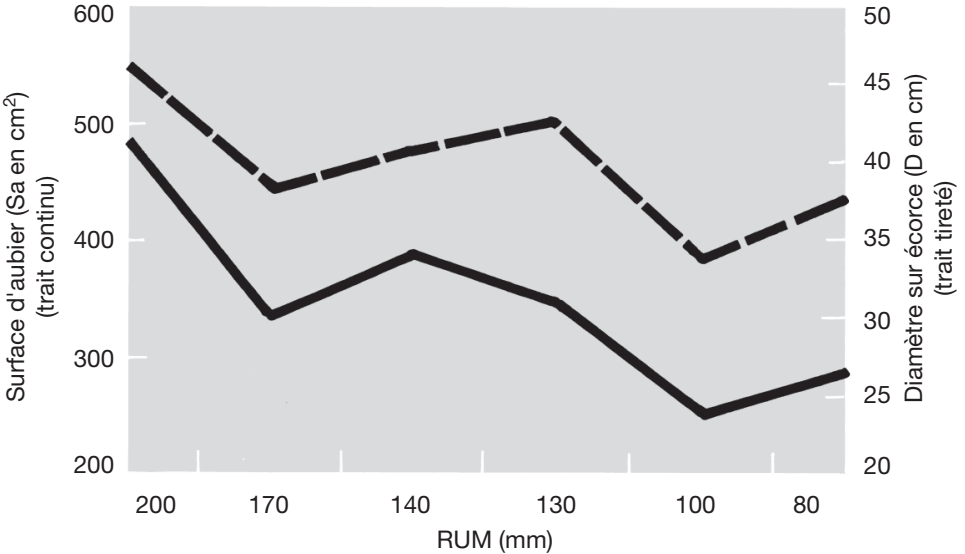

Chaque point est la moyenne d'au moins 10 arbres (8 placettes ; 144 arbres). 
À âge fixé, les arbres les plus vigoureux présentent une surface d'aubier plus importante. Pour les Chênes sessiles adultes de 80 ans, la surface d'aubier augmente de plus de 60 \% quand on passe d'un diamètre de $35 \mathrm{~cm}$ (surface d'aubier $=265 \mathrm{~cm}^{2}$ ) à un diamètre de $45 \mathrm{~cm}$ (surface d'aubier $=432 \mathrm{~cm}^{2}$ ). Pour les Chênes pédonculés de 70 ans, cet accroissement en diamètre correspond à une augmentation de près de $40 \%$ de la surface d'aubier $\left(248 \mathrm{~cm}^{2}\right.$ pour $35 \mathrm{~cm}$ et $338 \mathrm{~cm}^{2}$ pour $\left.45 \mathrm{~cm}\right)$. En revanche, pour les deux espèces, la proportion d'aubier dans le tronc reste constante, de l'ordre de $20 \%$ pour le Chêne sessile et $17 \%$ pour le Chêne pédonculé.

La prise en compte de la surface d'aubier et de la réserve utile maximale en eau confirme l'importance de ce paramètre dans la vigueur des arbres. Les arbres les plus vigoureux présentant des surfaces d'aubier supérieures sont localisés sur les stations bien alimentées en eau (figure 4, p. 532).

\section{Le pourcentage d'écorce}

Les résultats sont présentés dans le tableau VI (cidessous). Les deux espèces de chênes se distinguent nettement. Plus de $55 \%$ des Chênes pédonculés ont un pourcentage d'écorce supérieur à $15 \%$ alors que près de $60 \%$ des Chênes sessiles ont des valeurs inférieures à $15 \%$. Pour des diamètres compris entre 35 et $45 \mathrm{~cm}$, le pourcentage moyen d'écorce à 1,30 m est de l'ordre de 15,4\% pour le Chêne pédonculé et 13,3\% pour le Chêne sessile.

Tableau VI Pourcentage moyen d'écorce à $1,30 \mathrm{~m}$ selon la catégorie de diamètre sur écorce (entre 1 et 1,30 m) pour les Chênes sessile et pédonculé du réseau RENECOFOR

Le pourcentage d'écorce a été calculé selon le protocole du Centre technique du Bois et de l'Ameublement (CTBA) en faisant le rapport entre le diamètre mesuré sur écorce et le diamètre sous écorce calculé à partir de carottes de sondage prélevées au même niveau.

$\mathrm{n}=$ nombre d'arbres. Les valeurs du CTBA sont des moyennes obtenues à partir de plusieurs milliers de mesures effectuées dans les régions où le chêne est dominant. TSF = taillis-sous-futaie.

\begin{tabular}{|c|c|c|c|c|c|c|}
\hline \multirow{2}{*}{$\begin{array}{c}\text { Catégorie de } \\
\text { diamètre }(\mathrm{cm})\end{array}$} & \multicolumn{2}{|c|}{ Chêne sessile } & \multicolumn{2}{c|}{ Chêne pédonculé } & \multicolumn{2}{c|}{ Chênes (CTBA) } \\
\cline { 2 - 7 } & $\%$ & $\mathrm{n}$ & $\%$ & $\mathrm{n}$ & Futaie & TSF \\
\hline$<30 \ldots \ldots \ldots \ldots \ldots$ & 12,3 & 28 & 18,4 & 55 & - & - \\
{$[30-35] \ldots \ldots \ldots \ldots$} & 13,2 & 129 & 16,7 & 50 & 13,3 & 15,0 \\
{$[35-40] \ldots \ldots \ldots \ldots$} & 13,1 & 108 & 15,2 & 44 & - & - \\
] $40-45] \ldots \ldots \ldots \ldots$ & 13,6 & 163 & 15,7 & 34 & 12,9 & 14,7 \\
$>45 \ldots \ldots \ldots$ & 15,2 & 135 & 19,9 & 56 & - & - \\
$>50 \ldots \ldots \ldots \ldots \ldots$ & - & - & - & - & 12,4 & 13,1 \\
\hline
\end{tabular}




\section{F. LEBOURGEOIS}

\section{CONCLUSIONS}

Sans analyse poussée, l'étude dendrochronologique des placettes du réseau RENECOFOR fournit d'ores et déjà des données concrètes qui pourraient être utilisées dans les modèles de production et de gestion des peuplements étudiés. Les informations propres à chaque placette permettent de réorienter le forestier vers une gestion plus optimale du peuplement ou tout au moins plus conforme au plan local d'aménagement (cernes plus fins et plus réguliers, âge et diamètre d'exploitabilité des tiges, durée des révolutions). Pour le gestionnaire, des problèmes délicats peuvent néanmoins se poser quant aux scénarios sylvicoles à adopter et à leurs répercussions sur la qualité technologique du bois produit : comment gérer à (très) long terme un peuplement qui présente une forte hétérogénéité (âge, diamètre) ? Comment moduler le régime des coupes de façon à éviter une trop forte irrégularité des largeurs de cernes et une perte de qualité ?

À un niveau plus général, les données relatives à l'aubier, à l'écorce ou encore à l'anatomie des cernes permettent :

- de distinguer nettement les deux espèces de chênes,

- d'appréhender le fonctionnement physiologique des arbres (relation aubier et vigueur des tiges par exemple) ou la qualité de bois (variation des proportions des deux compartiments du cerne annuel selon le niveau de croissance ou l'âge cambial),

- de mieux "prédire" le volume de bois utilisable par l'exploitant (utilisation du pourcentage d'écorce et des proportions relatives d'aubier dans le tronc).

Au niveau stationnel, la qualité de l'alimentation en eau apparaît être un facteur important dans la vigueur des peuplements. À âge fixé, les arbres de plus gros diamètre, qui présentent des surfaces d'aubier importantes, sont localisés dans les stations à forte réserve utile en eau du sol. Actuellement, une analyse des relations cerne-climat est réalisée sur une partie des placettes du réseau. Cette étude devrait compléter les travaux présentés ici et mettre en évidence la part des conditions climatiques locales dans le déterminisme des variations de largeur des cernes et d'anatomie. À plus long terme, une étude détaillée des relations station-production pourrait être entreprise de façon à mettre en évidence les facteurs écologiques importants dans la productivité des chênaies du réseau.

\begin{tabular}{|c|}
\hline F. LEBOURGEOIS \\
Unité d'Écophysiologie forestière \\
Équipe Bioclimatologie \\
INRA \\
F-54280 CHAMPENOUX \\
Actuellement \\
Unité Écosystèmes forestiers \\
ÉCOLE NATIONALE DU GÉNIE RIRAL, \\
DES EAUX ET DES FORÊTS \\
14, rue Girardet \\
F-54042 NANCY CEDEX
\end{tabular}

\section{Remerciements}

L'auteur remercie particulièrement l'ensemble des responsables et suppléants locaux pour leur aide précieuse et efficace apportée lors de la récolte des données, ainsi que J.-F. Dhôte et A. Duquesnay pour leurs remarques constructives. 


\section{Biologie et forêt}

\section{BIBLIOGRAPHIE}

BECKER (M.), LÉVY (G.). - Croissance radiale comparée de chênes adultes (Quercus robur L. et Q. petraea (Matt.) Liebl.) sur sol hydromorphe acide : effet du drainage. - Acta Oecologica, Oecologia Plantarum, vol. 7 (21), n², 1986, pp. 121-143.

BECKER (M.), LÉVY (G.). - Le Dépérissement du Chêne. Les causes écologiques. Exemple de la forêt de Tronçais et premières conclusions. - Revue forestière française, vol. XXXV, $\mathrm{n}^{\circ}$ 5, 1983, pp. 341-356.

BRÉDA (N.), COCHARD (H.), DREYER (E.), GRANIER (A.). - Field comparison of transpiration, stomatal conductance and vulnerability to cavitation of Quercus petraea and Quercus robur under water stress. - Annales des Sciences forestières, vol. 50, n 6, 1993, pp. 529-632.

BRÉDA (N.), GRANIER (A.), AUSSENAC (G.). - Effects of thinning on soil and tree relations, transpiration and growth in an oak forest (Quercus petraea (Matt.) Liebl.). - Tree Physiology, vol. 15, 1994, pp. 295-306.

BRETTHES (A.), ULRICH (E.), Coordinateurs. - RENECOFOR - Caractéristiques pédologiques des cent deux peuplements du réseau. - Fontainebleau : Office national des Forêts - Département des Recherches techniques, 1997. - 573 p. (ISBN 2-84207-112-3).

CLUZEAU (C.), ULRICH (E.), LANIER (M.), GARNIER (F.). - RENECOFOR - Interprétation des mesures dendrométriques de 1991 à 1995 des 102 peuplements du réseau. - Fontainebleau : Office national des Forêts - Département des Recherches techniques, 1998. - 309 p.

COCHARD (H.), BRÉDA (N.), GRANIER (A.), AUSSENAC (G.). - Vulnerability to air embolism of three European oak species (Quercus petraea (Matt.) Liebl., Q. pubescens Willd, Q. robur L.). - Annales des Sciences forestières, vol. 49, 1992, pp. 225-233.

COCHARD (H.), TYREE (M.T.). - Xylem dysfunction in Quercus : vessel size, tyloses, cavitation and seasonal changes in embolism. - Tree Physiology, vol. 6, 1990, pp 393-407.

DERET-VARCIN (E.). - Étude comparative de la qualité du bois de trois types de Chênes (rouvres, pédonculés et intermédiaires) en forêt de Morimond. - Annales des Sciences forestières, vol. 40, $\mathrm{n}^{\circ} 3,1983$, pp. 373398.

DHÔTE (J.-F.). - Définition de scénarios d'éclaircie pour le Hêtre et le Chêne. - Revue forestière française, vol. XLVII, $\mathrm{n}^{\circ}$ spécial "Modélisation de la croissance des arbres forestiers et de la qualité des bois", 1995, pp. 106-110.

DHÔTE (J.-F.), HATSCH (E.), RITTIÉ (D.). - Profil de la tige et géométrie de l'aubier chez le chêne sessile (Quercus petraea (Matt.) Liebl.). - ONF - Bulletin technique, n³3, 1997, pp. 59-81.

DUPLAT (P.). - Sylviculture du Chêne pédonculé. - ONF - Bulletin technique, $\mathrm{n}^{\circ}$ 31, 1996, pp. $15-20$.

DUPOUEY (J.-L.). - Étude phytoécologique et écologique du massif forestier de Haguenau (Bas-Rhin). Apports méthodologiques. Potentialités sylvicoles. - INA-PG, 1983. - 140 p. (Thèse de Docteur-Ingénieur).

FEUILLAT (F.), DUPOUEY (J.-L.), SCIAMA (D.), KELLER (R.). - A new attempt at discrimination between Quercus petraea and Quercus robur based on wood anatomy. - Canadian Journal of Forest Research, vol. 27, 1997, pp. 343-351.

GRANIER (A.), ANFODILLO (T.), SABATTI (M.), COCHARD (H.), DREYER (E.), TOMASI (M.), VALENTINI (R.), BRÉDA (N.). - Axial and radial water flow in the trunk of oak trees : a quantitative and qualitative analysis. - Tree Physiology, vol. 14, 1994, pp. 1383-1396.

HATSCH (E.). - Répartition de l'aubier et acquisition de la forme de la tige chez le Chêne sessile (Quercus petraea (Matt.) Liebl.) : analyse, modélisation et relation avec le développement du houppier. - Nancy : ENGREF, 1997. - 183 p. (Thèse).

HUBER (F.). - Déterminisme de la surface des vaisseaux du bois de chênes indigènes (Quercus robur L., Quercus petraea Liebl.). Effet individuel, effet de l'appareil foliaire, des conditions climatiques et de l'âge de l'arbre. - Annales des Sciences forestières, vol. 50, n 5, 1993, pp. 509-524.

JARRET (P.). - Sylviculture du Chêne sessile. - ONF - Bulletin technique, n 31, 1996, pp. 20-28.

LEBOURGEOIS (F.). - RENECOFOR - Étude dendrochronologique des 102 peuplements du réseau. - Fontainebleau : Office national des Forêts - Département des Recherches techniques, 1997. - 307 p. (ISBN 2-84207-075-5).

LEBOURGEOIS (F.), ULRICH (E.), PONCE (R.). - Réactivité d'arbres âgés à l'ouverture du peuplement. Quelques exemples livrés par l'étude des placettes du réseau RENECOFOR. - Revue forestière française, vol $L, n^{\circ} 2$, 1998, pp. 139-148.

LÉVY (G.), BECKER (M.), DUHAMEL (D.). - A comparison of the ecology of pedunculate and sessile oaks : radial growth in the centre and northwest of France. - Forest Ecology and Management, vol. 55, 1992, pp. 51-63.

NEPVEU (G.). - Les Facteurs influençant la qualité du bois de Chêne (Chêne rouvre et Chêne pédonculé). Revue forestière française, vol. XLII, $\mathrm{n}^{\circ}$ 2, 1990, pp. 128-133.

NIEMINEM (T.M.). - Étude dendroécologique du Chêne (pédonculé et sessile) et du Hêtre dans une forêt de la plaine lorraine (forêt domaniale d'Amance). - 1988. - 40 p. (DEA de Biologie végétale et forestière).

ONF. Département des Recherches techniques. - Notice de présentation du Réseau national de suivi à long terme des Écosystèmes forestiers. - 1996. - 38 p. (ISBN 2-84207-021-6). 


\section{F. LEBOURGEOIS}

PARDÉ (J.), BOUCHON (J.). - Dendrométrie. - 2e édition. - Nancy : ENGREF, 1988. - 328 p.

PELLECUER (B.). - L'Écologie des chênes en forêt de Morimond et ses applications sylvicoles. - Université de Nancy I, 1976. - 173 p. (Thèse de Docteur-Ingénieur).

POLGE (H.). - Production de chênes de qualité en France. - Revue forestière française, vol. XXXVI, $n^{\circ}$ spécial "Dialogue forestier par dessus le Rhin", 1984, pp. 34-48.

POLGE (H.), KELLER (R.). - Qualité du bois et largeur d'accroissements en forêt de Tronçais. - Annales des Sciences forestières, vol. 30, $\mathrm{n}^{\circ}$ 2, 1973, pp. 91-125.

PONCE (R.), ULRICH (E.), GARNIER (F.). - RENECOFOR - Essai de synthèse de l'histoire des 102 peuplements du réseau. - Fontainebleau : Office national des Forêts - Département des Recherches techniques, 1998. 237 p. (ISBN 2-84207-136-6).

ULRICH (E.). - Le réseau RENECOFOR : objectifs et réalisation. - Revue forestière française, vol. XLVII, $n^{\circ} 2$, 1995, pp. 107-124.

ZHANG (S.Y.), NEPVEU (G.), OWOUNDI (R.E.). - Intratree and intertree variation in selected wood quality characteristics of European oak (Quercus petraea and Quercus robur). - Canadian Journal of Forest Research, vol. 24, 1994, pp. 1818-1823.

ZHANG (S.Y.), OWOUNDI (R.E.), NEPVEU (G.), MOTHE (F.), DHÔTE (J.-F.). - Modelling wood density in European oak (Quercus petraea and Quercus robur) and simulating the silvicultural influence. - Canadian Journal of Forest Research, vol. 23, 1993, pp. 2587-2593.

LES CHÊNES SESSILE ET PÉDONCULÉ (Quercus petraea Liebl. et Quercus robur L.) DANS LE RÉSEAU RENECOFOR : RYTHME DE CROISSANCE RADIALE, ANATOMIE DU BOIS, DE L'AUBIER ET DE L'ÉCORCE (Résumé)

Les objectifs de cet article sont d'analyser les rythmes de croissance radiale des 30 chênaies du réseau RENECOFOR (614 chênes sessiles et 263 chênes pédonculés) et de comparer les deux espèces quant aux caractéristiques du bois initial et du bois final dans le cerne annuel, de l'aubier et de l'écorce. La comparaison des deux espèces montre que :

- le pourcentage de bois initial dans le cerne annuel augmente des jeunes cernes (10 ans) aux cernes âgés (> 70 ans) dans des proportions de 20 à $30-35 \%$ pour le Chêne sessile et $40-45 \%$ pour le Chêne pédonculé ;

- à âge de cerne fixé (40-60 ans), une augmentation de la largeur du cerne de 1 à $3 \mathrm{~mm}$ se traduit par une augmentation relative de la proportion de bois final dans le cerne de $23 \%$ pour le Chêne sessile et $35 \%$ pour le Chêne pédonculé ;

- l'aubier du Chêne pédonculé est de 15 à 20 \% moins large que l'aubier du Chêne sessile en relation avec un nombre moyen de cernes dans l'aubier plus faible ;

- pour des diamètres compris entre 35 et $45 \mathrm{~cm}$, le pourcentage moyen d'écorce à 1,30 m est de l'ordre de $15,4 \%$ pour le Chêne pédonculé et $13,3 \%$ pour le Chêne sessile.

Ces différences sont discutées en terme de fonctionnement physiologique des arbres et de la qualité de bois.

\section{THE SESSILE AND THE PEDUNCULATE OAK (Quercus petraea Liebl. and Quercus robur L.) IN THE RENE- COFOR NETWORK : RADIAL GROWTH RATE AND WOOD, SAPWOOD AND BARK ANATOMY (Abstract)}

The purpose of this article is to analyse the rate of radial growth in the 30 oak groves that make up the RENECOFOR network (614 sessiles and 263 pedunculates) and compare the early and late wood in the growth layer for the two species and their sapwood and bark. The comparison shows that :

- the percentage of early wood in the growth layer increases in rings from the age of 10 to more than 70 in proportions ranging from 20 to $30-35 \%$ for the sessile oak and $40-45 \%$ for the pedunculate oak;

- at a set ring age (40-60 years) a 1 to $3 \mathrm{~mm}$ increase in width is reflected in an relative increase of late wood in the ring - $23 \%$ for the sessile oak and $35 \%$ for the pedunculate;

- sapwood in the pedunculate oak is 15 to $20 \%$ narrower than in the sessile associated with a lower mean number of rings in the sapwood;

- for diameter ranging between 35 and $45 \mathrm{~cm}$, the mean percentage of bark at 1.30 meters is in the range of $15.4 \%$ for the pedunculate and $13.3 \%$ for the sessile.

These differences are discussed in terms of the physiology of the trees and the quality of wood. 